\title{
Abrus agglutinin suppresses human hepatocellular carcinoma in vitro and in vivo by inducing caspase- mediated cell death
}

\author{
Subhadip MUKHOPADHYAY ${ }^{1}$, Prashanta Kumar PANDA ${ }^{1}$, Durgesh Nandini DAS ${ }^{1}$, Niharika SINHA ${ }^{1}$, Birendra BEHERA ${ }^{2}$, \\ Tapas Kumar MAITI', Sujit Kumar BHUTIA ${ }^{1, *}$ \\ ${ }^{1}$ Department of Life Science, National Institute of Technology, Rourkela, Odisha, India; ${ }^{2}$ Department of Biotechnology, Indian Institute \\ of Technology, Kharagpur, West Bengal, India
}

Aim: Abrus agglutinin (AGG) from the seeds of Indian medicinal plant Abrus precatorius belongs to the class II ribosome inactivating protein family. In this study we investigated the anticancer effects of AGG against human hepatocellular carcinoma in vitro and in vivo. Methods: Cell proliferation, DNA fragmentation, Annexin V binding, immunocytofluorescence, Western blotting, caspase activity assays and luciferase assays were performed to evaluate AGG in human liver cancer cells HepG2. Immunohistochemical staining and TUNEL expression were studied in tumor samples of HepG2-xenografted nude mice.

Results: AGG induced apoptosis in HepG2 cells in a dose- and time-dependent manner. AGG-treated HepG2 cells demonstrated an increase in caspase 3/7, 8 and 9 activities and a sharp decrease in the Bcl-2/Bax ratio, indicating activation of a caspase cascade. Co-treatment of HepG2 cells with AGG and a caspase inhibitor or treatment of AGG in Bax knockout HepG2 cells decreased the caspase 3/7 activity in comparison to HepG2 cells exposed only to AGG. Moreover, AGG decreased the expression of Hsp90 and suppressed Akt phosphorylation and NF-kB expression in HepG2 cells. Finally, AGG treatment significantly reduced tumor growth in nude mice bearing HepG2 xenografts, increased TUNEL expression and decreased CD-31 and Ki-67 expression compared to levels observed in the untreated control mice bearing HepG2 cells.

Conclusion: AGG inhibits the growth and progression of HepG2 cells by inducing caspase-mediated cell death. The agglutinin could be an alternative natural remedy for the treatment of human hepatocellular carcinomas.

Keywords: anticancer drug; Abrus agglutinin; hepatocellular carcinoma; apoptosis; caspase; Akt; Hsp90, NF-kB; HepG2 xenografts

Acta Pharmacologica Sinica (2014) 35: 814-824; doi: 10.1038/aps.2014.15; published online 5 May 2014

\section{Introduction}

Hepatocellular carcinoma (HCC) poses a major global health risk, killing more than half a million people each year ${ }^{[1]}$. HCC is the fifth most common cause of cancer and the third leading cause of cancer-related deaths worldwide ${ }^{[2,3]}$. Although the worldwide distribution of HCC incidences varies largely due to different etiological factors, its occurrence is greater among Asian and African countries compared to western nations ${ }^{[4]}$. The HCC prevalence and mortality continue to increase, predominantly among men ${ }^{[5,6]}$. Despite several advancements in screening techniques and various types of drugs, HCC relapse is frequent and plays a major role in limiting patients' survival. A natural molecule targeting HCC could be a successful

\footnotetext{
* To whom correspondence should be addressed.

E-mail sujitb@nitrkl.ac.in, bhutiask@gmail.com

Received 2013-07-26 Accepted 2014-01-04
}

next-generation cancer treatment. In this regard, there has been a significant increase in the use of plant lectins as alternative therapeutic molecules owing to their high specificity, nontoxic nature and availability.

The plant lectin Abrus agglutinin (AGG), which is isolated from the seeds of the medicinal Indian plant Abrus precatorius, is a hetero-dimeric glycoprotein with a molecular weight of $134 \mathrm{kDa}$ and a high specificity for Gal $(\beta 1 \rightarrow 3)$ GalNAc $^{[7]}$. AGG belongs to the class II ribosome inactivating protein (RIP) family and comprises two $30 \mathrm{kDa}$ toxic A chain subunits and two $31 \mathrm{kDa}$ galactose-binding B chain subunits, which are interconnected via a single disulfide bond ${ }^{[8,9]}$. AGG has a protein synthesis inhibitory concentration $\left(\mathrm{IC}_{50}\right)$ of $0.469 \mu \mathrm{g} / \mathrm{mL}$ and a lethal dose $\left(\mathrm{LD}_{50}\right)$ of $5 \mathrm{mg} / \mathrm{kg}$ body weight in mice ${ }^{[9]}$.

Previous work from our group established the proapoptotic, antitumor effect of AGG in several tumor models at sub-lethal doses, demonstrating that AGG activity is highly specific for 
cancerous cells and that it induces cell death by targeting mitochondrial apoptosis ${ }^{[7,9]}$. In addition to its antineoplastic potential, AGG elicits both humoral and cellular immunity in normal as well as tumor-bearing mice. AGG also activates splenocytes, stimulating a Th1-directed immune response. Interestingly, under heated denaturing conditions, AGG, as well as its peptides, shows peritoneal macrophage- and NK cell-stimulating properties in mouse splenocytes in addition to a significant apoptosis-mediated reduction in Dalton's lymphoma tumors ${ }^{[10-14]}$.

In this study, we report for the first time the in vitro and in vivo growth inhibitory potential of AGG on HepG2 cells and xenografts in athymic nude mice with the aim of evaluating the role of apoptosis stimulation as a fundamental mechanism in its anticancer activity. We examined various parameters of apoptosis, including Bax upregulation, Bcl-2 downregulation, poly (ADP ribose) polymerase (PARP) cleavage, phosphrylated-Akt (p-Akt), NF-kB and Hsp90 reduction and in vitro DNA fragmentation, together with an analysis of Ki-67 and CD-31 by immunohistochemistry and TUNEL assays using nude mice bearing HepG2 xenografts.

\section{Materials and methods Reagents}

3-(4,5-dimethylthiazol-2-yl)-2,5-diphenyltetrazolium (MTT), 4',6-diamidino-2-phenylindole dihydrochloride (DAPI), dimethylsulfoxide (DMSO), propidium iodide (PI), trypsin and agarose were purchased from Sigma-Aldrich (St Louis, MO, USA). Fetal bovine serum (sterile-filtered, South American origin), minimal essential medium (MEM), Dulbecco's modified Eagle medium (DMEM) and antibiotic-antimycotic $(100 \times)$ solution were purchased from Invitrogen (Carlsbad, CA, USA). Caspase-3 inhibitor III (Ac-DEVD-CMK) was purchased from Calbiochem (San Diego, CA, USA). Other reagents were of analytical grade or of the highest quality available.

\section{Abrus agglutinin}

AGG was isolated and purified from Abrus precatorius seeds by ammonium sulfate fractionation followed by lactamyl sepharose affinity chromatography and Sephadex G-100 gel permeation chromatography ${ }^{[8]}$. The activity of isolated AGG was measured by a hemagglutination assay, and the purity of the protein was subsequently analyzed by SDS-PAGE, nativePAGE and gel permeation in HPLC.

\section{Cell culture}

The human liver cancer cells HepG2 and the immortalized human keratinocyte cells $\mathrm{HaCaT}$ were obtained from the National Centre of Cell Science, Pune. HepG2 and HaCaT cells were cultured in MEM and DMEM respectively, complemented with $10 \%$ fetal bovine serum and antibiotic-antimycotic solution followed by incubation at $37^{\circ} \mathrm{C}$ in a humidified ( $95 \%$ air : $5 \% \mathrm{CO}_{2}$ ) incubator.

\section{Assay for cell viability}

HepG2 and HaCaT cells were harvested from maintenance cultures during the logarithmic phase after being counted in a hemocytometer using trypan blue solution. The cell concentrations were adjusted to $5 \times 10^{4}$ cells $/ \mathrm{mL}$, and the cells were plated in 96-well flat-bottom culture plates and incubated with various concentrations of AGG for different durations. The effect of AGG on cancer cell viability was studied using a MTT dye reduction assay by measuring the optical density at 595 $\mathrm{nm}$ using a micro-plate reader spectrophotometer (PerkinElmer, Walthman, MA, USA $)^{[7]}$. Phosphate buffered saline (PBS) that was used to dissolve AGG was used in the control treatment.

\section{DAPI staining of HepG2 cells}

Nuclear staining using the DAPI stain was performed according to the method previously described ${ }^{[9]}$. In brief, HepG2 cells that were either untreated or treated with AGG were smeared on a clean glass slide, and the cells were fixed with $3.7 \%$ paraformaldehyde for $15 \mathrm{~min}$, permeabilized with $0.1 \%$ Triton $\mathrm{X}-100$ and stained with $1 \mu \mathrm{g} / \mathrm{mL}$ DAPI for $5 \mathrm{~min}$ at $37^{\circ} \mathrm{C}$. The cells were then washed with PBS and examined by inverted fluorescence microscopy (Olympus IX 71, Tokyo, Japan).

\section{Clonogenic survival determination in HepG2 cells}

The effect of AGG treatment on the reproductive potential of HepG2 cells was assessed using the colony formation assay. Briefly, HepG2 cells were treated with different concentrations of AGG for $12 \mathrm{~h}$ and collected by trypsinization. The cells were counted and replated in triplicate on a 6-well tissue culture plate with 3000 cells/well. The cells were cultured for 14 days, with the growth medium being replaced every 3 days. The cells were then stained with $0.5 \%$ crystal violet (in methanol/water, 1:1), and the colonies were counted ${ }^{[15]}$.

\section{DNA fragmentation assay in AGG-treated HepG2 cells}

For the DNA fragmentation assay, a non-enzymatic method was adopted ${ }^{[16]}$. Briefly, two million HepG2 cells were seeded onto a $60-\mathrm{mm}$ Petri dish and treated with different concentrations of AGG. After $24 \mathrm{~h}$, the cells were dislodged and pelleted down. Then, $100 \mu \mathrm{L}$ DMSO was added to the pellets and mixed well, followed by the addition of an equal amount of TE buffer ( $\mathrm{pH} 7.4)$ with $2 \%$ SDS. The mixture was then vortexed and centrifuged at $12000 \times g$ at $4^{\circ} \mathrm{C}$, and $40 \mu \mathrm{L}$ from the resulting supernatant was then loaded onto a 1.5\% agarose gel. The DNA was stained by ethidium bromide and visualized in a Bio-Rad gel documentation system using the Quantity One 1-D analysis software (version 4.6.9, Bio-Rad Laboratories, Inc., Hercules, CA, USA).

\section{Annexin $\mathrm{V}$ binding}

HepG2 cells were treated with different concentrations of AGG, after which the cell pellets were collected after trypsinization. The pellets were washed twice with PBS by centrifu- 
gation (Hitachi CR 22GIII High Speed Refrigerated Centrifuge, Tokyo, Japan) at $210 \times g$ for $5 \mathrm{~min}$ at room temperature. After removal of supernatant, the cells were incubated with Annexin $\mathrm{V}$ and propidium iodide in binding buffer and analyzed by flow cytometry (BD Biosciences, Franklin Lakes, NJ, USA) ${ }^{[17]}$.

\section{Plasmids and transfections}

HepG2 cells were cultured in $60-\mathrm{mm}$ petri plates and transfected at $80 \%$ confluence with Lipofectamine 2000 reagent (Invitrogen) in Opti-MEM (Invitrogen) following the manufacturer's protocol. The transfection was performed in the presence of a specific human Bax knockout $(\mathrm{KO})$ vector (Addgene plasmid 16575) ${ }^{[18]}$ and an empty backbone pcDNA3 plasmid (Addgene plasmid 10792), which was used for mock transfection. The cells were analyzed $48 \mathrm{~h}$ after transfection.

\section{NF-KB luciferase assay}

HepG2 cells were seeded into 24-well cultured plates and transfected with the pNFkB-Luc vector (BD Biosciences) and Renilla pGL4.74 [hRluc/TK] vector (Promega, Madison, WI, USA), as discussed in previous section on plasmids and transfections. The cells were treated with varied concentrations of AGG for $12 \mathrm{~h}$, and cell lysates were prepared using Passive Lysis Buffer (Promega). The luminescence was then quantified using a Dual-Luciferase Reporter Assay (E1910; Promega) and a FLUOstar Optima Luminometer (BMG Labtech, Durham, NC, USA). The Relative Light Units (RLU) were obtained for both the Renilla and Firefly Luciferases, and all of the results were expressed as the ratio of Luciferase RLU to Renilla RLU ${ }^{[19]}$.

\section{Caspase assays}

HepG2 cells were seeded onto 6-well plates and treated with AGG for $24 \mathrm{~h}$. Then, the cell lysates were used to measure the caspase activity using the Caspase-Glo assay following the manufacturer's protocol (Promega).

\section{Immunocytofluorescence staining and analysis}

HepG2 cells were seeded onto chamber slides (Falcon, Becton Dickinson, Franklin Lakes, NJ, USA) and maintained in MEM with $10 \%$ fetal bovine serum. Approximately $24 \mathrm{~h}$ after the AGG treatment at the mentioned doses, the cells were fixed with $2 \%$ paraformaldehyde, permeabilized by $0.1 \%$ Triton $X-100$, and then incubated with the primary antibodies for NF-KB (1:50; Santa Cruz, Dallas, TX, USA) and Hsp90 (1:50; Santa Cruz). The p-Akt and total Akt (1:500; Cell Signaling Technology, Danvers, MA, USA) expression variation was studied at the indicated durations of AGG $(10 \mu \mathrm{g} / \mathrm{mL})$ exposure. The secondary anti-rabbit and/or anti-mouse antibodies conjugated with Alexa Fluor (Life Technologies, Grand Island, NY, USA) were used to study the fluorescence of our desired proteins, which were detected using an Olympus IX71 fluorescent inverted microscope and the cellSens Standard software (version 1.6, Olympus Soft Imaging Solutions GmbH, JohannKrane-Weg, Münster, Germany $)^{[15]}$. The immunocytofluorescent images were quantified by measuring the integrated optical density and area fraction with the ImageJ software (National Institute of Mental Health, Bethesda, MA, USA) ${ }^{[20]}$.

\section{Western blotting analysis}

HepG2 cells were treated with different doses of AGG, followed by the extraction of proteins. A lysis buffer containing $50 \mathrm{mmol} / \mathrm{L}$ Tris- $\mathrm{HCl}$ (pH 7.6), $25 \mathrm{mmol} / \mathrm{L} \mathrm{NaCl}, 0.5 \%$ Triton $\mathrm{X}-100$ and $2 \mathrm{mmol} / \mathrm{L}$ dithiothreitol was used to extract the cytosolic proteins. The extraction buffer was supplemented on the day of use with $1 \times$ cocktail protease inhibitors (Roche Applied Science, Indianapolis, IN, USA), $1 \mathrm{mmol} / \mathrm{L}$ phenylmethylsulfonyl fluoride, $10 \mathrm{mmol} / \mathrm{L}$ sodium fluoride and 1 $\mathrm{mmol} / \mathrm{L}$ sodium orthovanadate. Approximately $50 \mu \mathrm{g}$ protein was subjected to electrophoresis through the distinct percentages of SDS polyacrylamide gel, followed by the transfer of proteins onto polyvinylidene difluoride membranes. The membranes were blocked with a buffer containing $5 \%$ BSA in PBST at room temperature for $1 \mathrm{~h}$ and subsequently incubated in the same buffer containing various primary antibodies, including PARP, Bcl-2, Bax (1:1000; Cell Signaling Technology) and Hsp90 (1:50; Santa Cruz). The membranes were then incubated at room temperature for $1 \mathrm{~h}$ with anti-rabbit and/ or anti-mouse antibodies conjugated with horseradish peroxidase. The proteins of interest were detected using the chemiluminescence method (Perkin-Elmer Life Sciences, Boston, MA, USA $)^{[15,17]}$. The protein band intensities from the Western blots were quantified using the ImageJ software ${ }^{[20]}$.

\section{Human liver cancer xenografts in athymic nude mice}

Male athymic nude mice ( $25 \pm 2 \mathrm{~g}$, 6-8 weeks old) were used for the anticancer study. The mice were housed in open-top polysulfone cages and provided food and water ad libitum. Room temperature was maintained at $22 \pm 2{ }^{\circ} \mathrm{C}$ with a light and dark cycle of $14 / 10 \mathrm{~h}$. All of the animal experiments were performed according to the rules of the "Committee for the purpose of control and supervision of experiments on animals, Ministry of Environment and Forests, Government of India" and the Institutional Animal Ethics Committee, Indian Institute of Technology, Kharagpur, Pin-721302. The HepG2 cells $\left(2 \times 10^{6}\right)$ in $100 \mu \mathrm{L}$ PBS were subcutaneously injected in the flank of male athymic nude mice. After the establishment of visible tumors of $\sim 100 \mathrm{~mm}^{3}$, which required approximately 10-15 days, AGG (500 $\mu \mathrm{g} / \mathrm{kg}$ body weight) was intraperitoneally injected for next ten consecutive days. A minimum of five animals were used per experimental condition. The tumor volume was measured twice weekly using a caliper and calculated using the formula $\Pi / 6 \times$ larger diameter $\times$ (smaller diameter $)^{2}$. At the end of the experiment, the animals were sacrificed, and the tumors were removed and weighed ${ }^{[21]}$.

\section{Immunohistochemical staining}

For the immunohistochemical analysis, formalin-fixed and paraffin-embedded specimens of 3-4 mm thickness were sectioned. The sections were deparaffinized, re-hydrated and quenched in $3 \% \mathrm{H}_{2} \mathrm{O}_{2}$ for $20 \mathrm{~min}$. The sections were washed with PBS and blocked in PBS containing 1\% BSA for 20 min at 
$37^{\circ} \mathrm{C}$. The sections were incubated in monoclonal anti-CD-31 (1:200; Dako Corporation, Carpenteria, CA, USA), anti-Ki-67 (1:50; BD Pharmingen, San Diego, CA, USA) or anti-TUNEL solutions (1:50; Roche, Indianapolis, IN, USA) overnight at $4^{\circ} \mathrm{C}$, washed thrice in PBS, incubated with an avidin-biotinperoxidase complex (VECTASTAIN Elite ABC Kit; Vector Laboratories, Burlingame, CA, USA) and then washed twice in PBS. The immunoreactivity was determined using diaminobenzidine as the final chromogen. Finally, the sections were counterstained with Mayer's hematoxylin, dehydrated through a sequence of increasing concentrations of alcohol, cleared in xylene and mounted with epoxydic medium. During the immunohistochemical assay, the proof slides were coupled with negative control slides, from which the primary antibody was omitted. The sections were also processed for hematoxylin and eosin staining ${ }^{[21]}$.

\section{Statistical analysis}

All data are presented as the mean \pm SD. The experimental results were analyzed using Student's $t$-test. $P<0.05$ was considered the level of significance when comparing the values from the experimental treatments to those of the control.

\section{Results}

AGG treatment resulted in a significant inhibition of cell growth and clonogenic cell survival

In an initial study, we evaluated the effect of AGG on the survival ability of HepG2, liver carcinoma cell line and non-tumor human keratinocyte cell line HaCaT. After AGG treatment at different concentrations for 24,48 and $72 \mathrm{~h}$, the survival of HepG2 cells was decreased in a dose-dependent and timedependent pattern, whereas the cell proliferation of HaCaT cells was relatively unaffected (Figure 1A). Our data demonstrate that AGG is highly specific in inhibiting the growth and proliferation of HepG2 cells but not for normal cells. From the colony-forming assay, we determined the ability of AGGtreated carcinoma cells to grow into colonies after the infinite divisions of the cells. The data demonstrated that AGG significantly decreased the colony-forming potential of liver cancer cells in a dose-dependent manner. The colony numbers decreased from $180 \pm 28$ in the control group to $140 \pm 18,76 \pm 11$ and $50 \pm 7$ in the groups treated with AGG concentrations of 0.1, 1.0 and $10 \mu \mathrm{g} / \mathrm{mL}$, respectively (Figure 1B).

\section{AGG induces apoptosis in HepG2 cells}

Next, we explored how AGG treatment prevents HepG2 cell growth and proliferation by inducing apoptosis. To validate our findings, we performed the flow cytometric analysis using Annexin V/PI staining. A sharp increase in the cellular population undergoing apoptosis was observed in the AGG-treated HepG2 cells compared to the control group. The apoptotic population (which included early, late apoptotic and secondary necrotic cells) increased from $6 \% \pm 0.6 \%$ in the control group to $11 \% \pm 1.7 \%, 22 \% \pm 3.4 \%$ and $44 \% \pm 4.5 \%$ in the groups treated with AGG concentrations of $0.1,1.0$ and $10 \mu \mathrm{g} / \mathrm{mL}$, respectively (Figure 2A; Supplementary Figure 1A). Further-
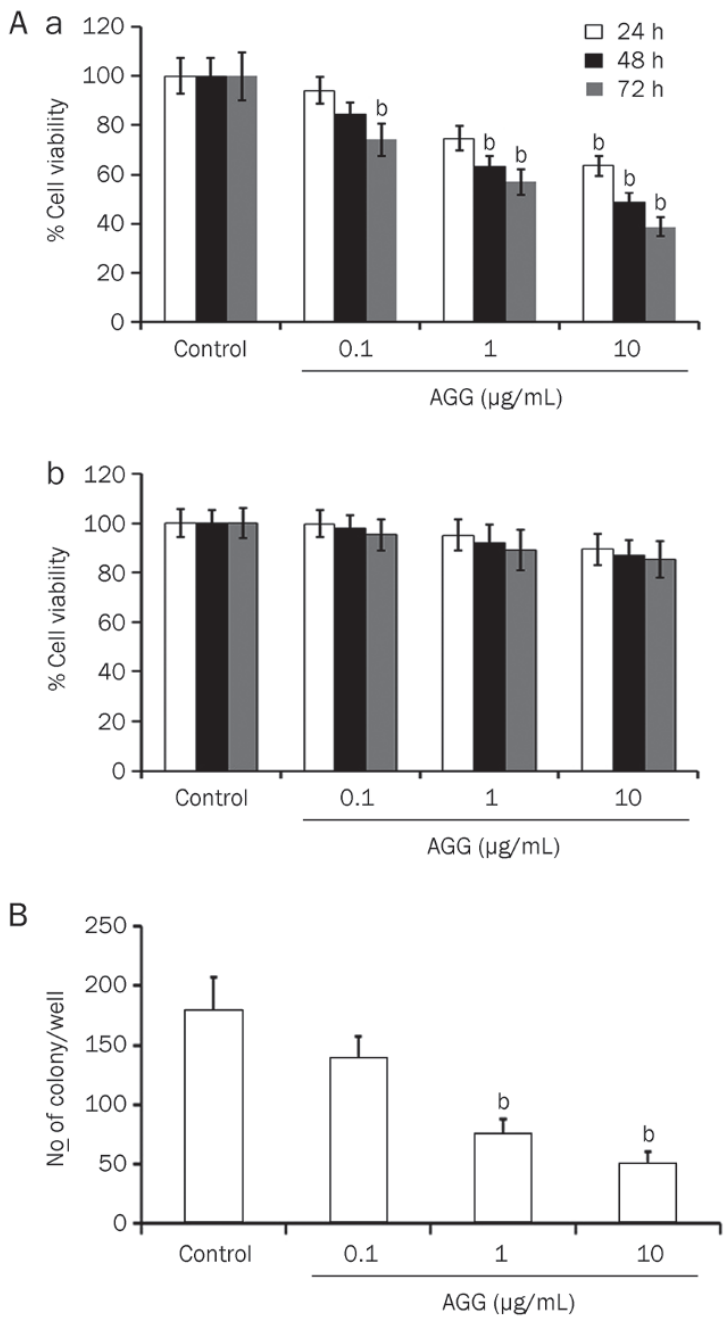

Figure 1. Effect of AGG on cell viability and growth. (A) HepG2 (a) and $\mathrm{HaCaT}$ cells (b) were treated with AGG at the indicated concentrations for 24,48 and $72 \mathrm{~h}$ with six replicates in each concentration. Cell viability was measured using a MTT assay. Data are reported as the mean \pm SD of six different observations and compared to the PBS control using Student's t-test. (B) After $12 \mathrm{~h}$ of treatment with AGG, HepG2 cells were processed as described in the 'Methods'. The colonies that formed were stained with $0.5 \%$ crystal violet (in methanol/water, 1:1) and counted. ${ }^{\mathrm{b}} \mathrm{P}<0.05$ vs control.

more, DAPI staining of the AGG-treated HepG2 cells showed characteristic apoptotic morphology, including nuclear membrane blebbing with condensed chromatin, cellular shrinkage and the formation of apoptotic bodies (Figure 2B). The typical apoptotic laddering pattern, which is a characteristic biochemical hallmark of apoptosis, was detected via agarose gel electrophoresis in HepG2 cells after $24 \mathrm{~h}$ of exposure to AGG at different concentrations (Figure 2C). We next analyzed protein expression by Western blotting to identify the molecules that were triggered in AGG-induced apoptosis. We observed a clear decrease in the level of antiapoptotic protein Bcl-2 expression in a dose-dependent manner, whereas the level of proapoptotic protein Bax expression concomitantly increased. 
A

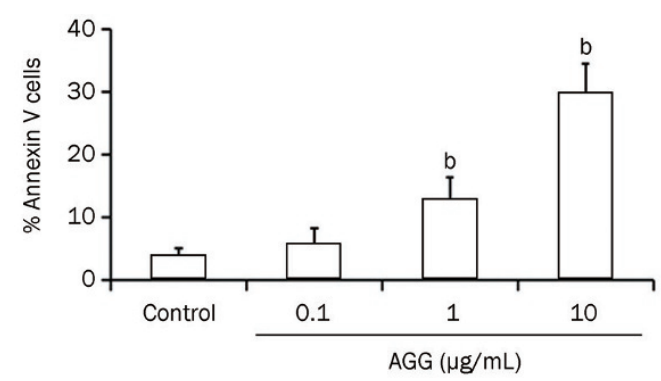

B
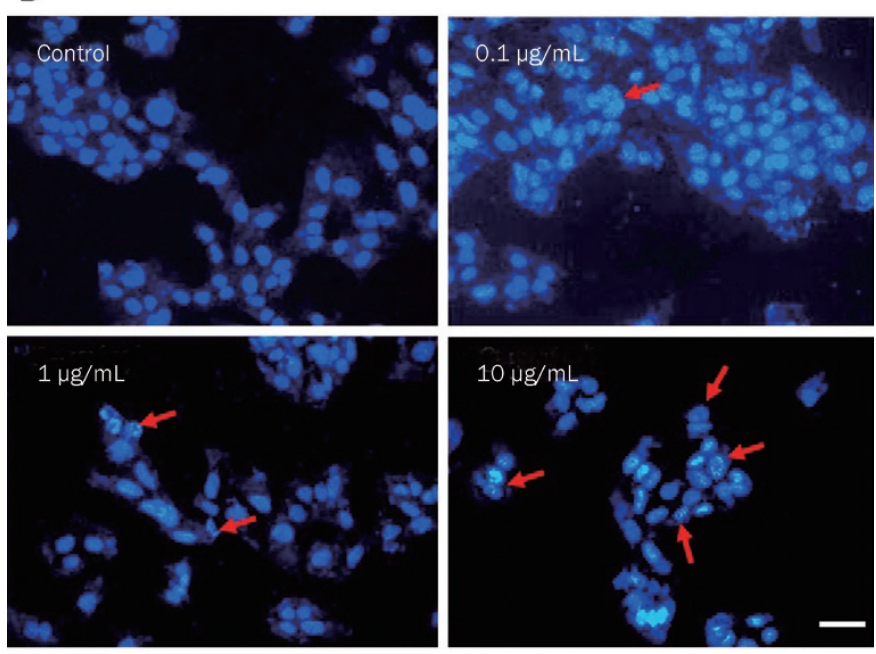

C

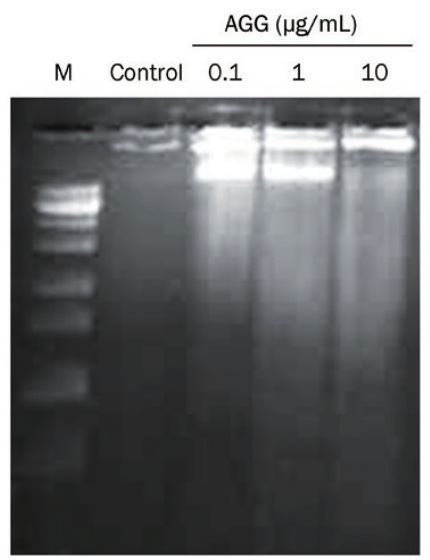

Figure 2. Effect of AGG on apoptosis induction in HepG2 cells. (A) HepG2 cells were treated with different concentrations $(0.1,1.0$ and $10 \mu \mathrm{g} / \mathrm{mL})$ of AGG for $24 \mathrm{~h}$ and then assayed for the \% Annexin V-positive cells. ${ }^{\mathrm{b}} \mathrm{P}<0.05$ vs control. (B) The morphological changes in the nuclei of HepG2 cells between the control and treated groups were studied after fixing the cells with $3.7 \%$ paraformaldehyde for 15 min, permeabilizing with $0.1 \%$ Triton $\mathrm{X}-100$ and staining with $1 \mathrm{\mu g} / \mathrm{mL}$ DAPI for $5 \mathrm{~min}$ at $37^{\circ} \mathrm{C}$. The cells were then washed with PBS and examined by fluorescence microscopy (Olympus IX71, 200x). The arrow marks indicate condensed and fragmented nuclei in the abovementioned dose-treated groups. The scale bar represents $50 \mu \mathrm{m}$. (C) Twenty-four hours after the AGG treatments at the indicated doses, the HepG2 cells were analyzed for DNA fragmentation by ethidium bromide staining ('M' indicates DNA ladder).
Thereby, there was a dose-dependent increase in the Bax/Bcl-2 ratio leading to apoptosis (Figure 3A-3C). PARP-1 is regularly involved in DNA damage repair by the addition of poly (ADP ribose) polymers as an adaptation to a variety of cellular stresses $^{[22]}$. PARP-1 cleavage is a signature motif of protease action that is unique to the apoptotic death program. The expression level of the PARP-1 cleavage fragment was greater in the AGG-treated HepG2 cells (Figure 3A and 3D). In addition, we measured the activity of caspase $3 / 7,8$ and 9 in AGGtreated HepG2 cells through caspase Glo assay and found that the caspase activity levels increased in a dose-dependent manner (Figure 3E). This finding indicates that AGG is able to induce both intrinsic and extrinsic apoptosis; specifically, AGG inhibits the growth of HepG2 cells through caspasedependent apoptosis. To support this finding, we inhibited caspase 3 using Ac-DEVD-CMK and observed a reduction in the caspase 3/7 activity level in the AGG/Ac-DEVD-CMK/ HepG2 cells compared to AGG/HepG2 cells (Figure 3F). This result strongly suggests that AGG induces caspase-mediated cell death in HepG2 cells. Moreover, to demonstrate the effect of AGG on the growth and progression of HepG2 cells after caspase inhibition, we performed a cell viability assay to evaluate whether AGG/Ac-DEVD-CMK/HepG2 cells have significantly higher growth and proliferation than AGG/HepG2 cells (Figure 3G). Furthermore, we also investigated the effect of Bax knockdown on the apoptotic potential of AGG in HepG2 cells using a Bax KO plasmid (Supplementary Figure $1 \mathrm{~B}$ and $1 \mathrm{C})$. There was a significant decrease in the level of caspase 3/7 activity in the AGG-treated Bax KO/HepG2 cells compared to the AGG-treated mock-transfected HepG2 cells (using the empty backbone pcDNA3 plasmid) (Figure $3 \mathrm{H}$ ). This result indicates that AGG induces an increase in proapoptotic Bax expression, thereby activating key molecules, including caspase 3 , which subsequently directs the ultimate process of programmed type I cell death.

\section{AGG downregulates the expression of Hsp90, Akt and NF-KB in HepG2 cells}

$\mathrm{NF}-\mathrm{KB}$ is involved in regulating the development and maturation of malignancy. NF-kB has frequently been reported to be aberrantly or constitutively expressed in human cancers, impeding apoptosis and promoting cellular migration, proliferation and chemoresistance. NF-KB has recently become a prime target for pharmaceutical research ${ }^{[23]}$. Our study demonstrated that the expression level of NF-kB (p50) in AGGtreated HepG2 cells decreased in a dose-dependent manner, as shown by immunocytofluorescence (Figure 4A and 4B). Moreover, NF-KB expression was also remarkably decreased at the transcriptional level, as demonstrated by Dual-Luciferase Reporter Assay (Figure 4C). Although further study is required to unravel the specific molecular mechanism, interestingly, the initial data show that AGG represses Hsp90 signaling along with p-Akt expression, thereby inducing a decrease in NF-KB expression. The data also demonstrate that 
A

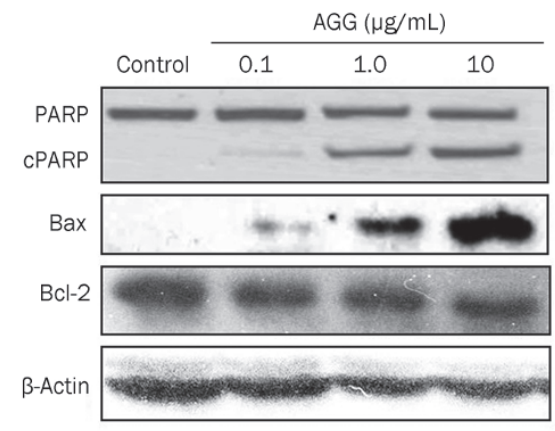

C

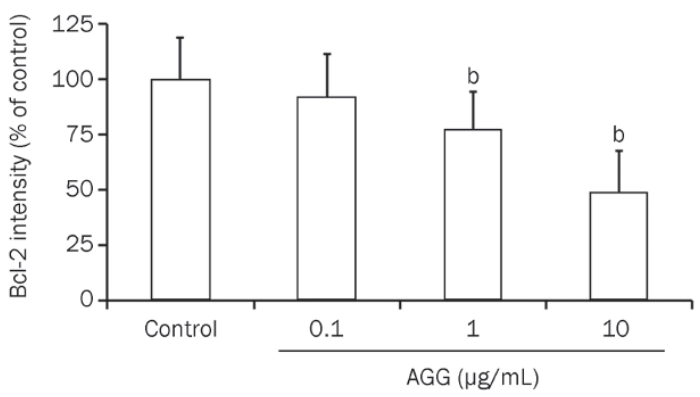

E

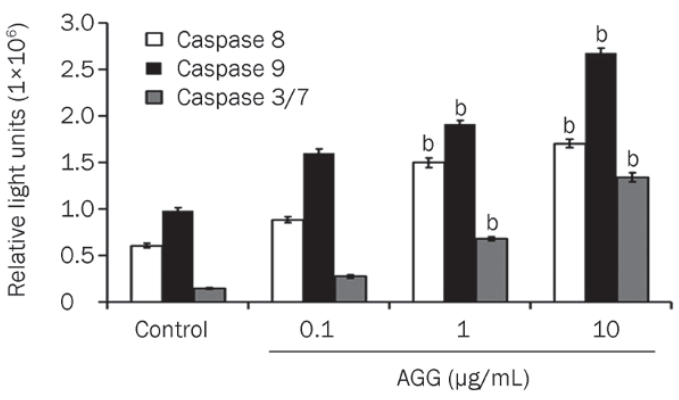

G

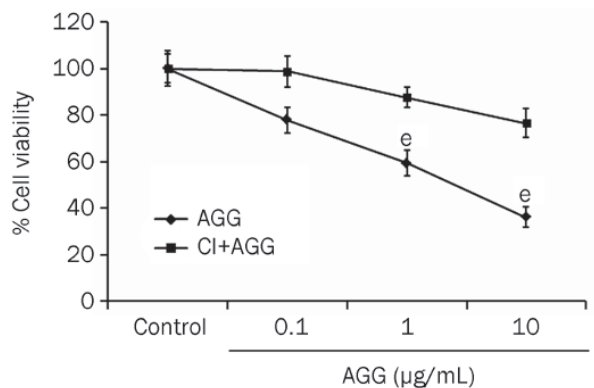

B

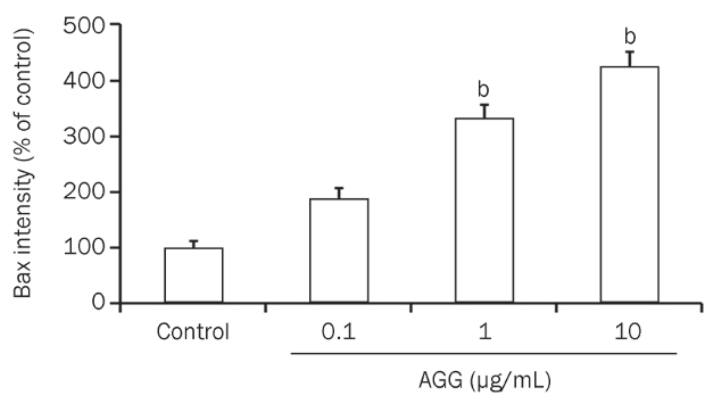

D

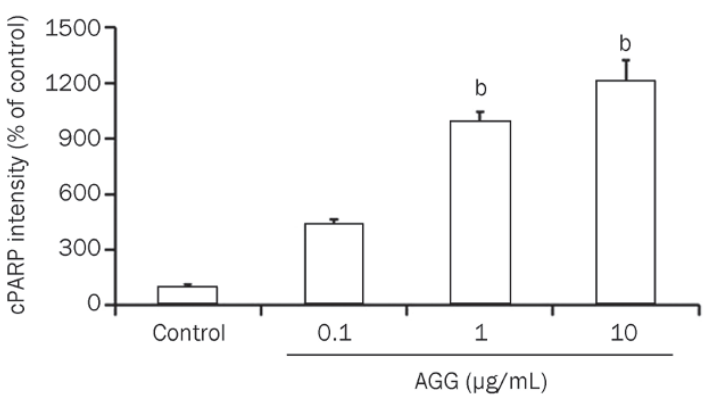

$\mathrm{F}$

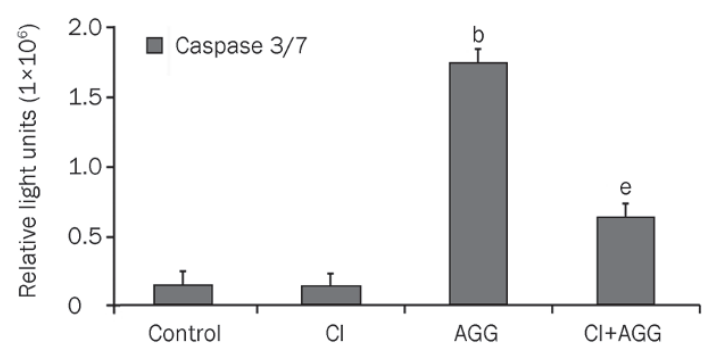

$\mathrm{H}$

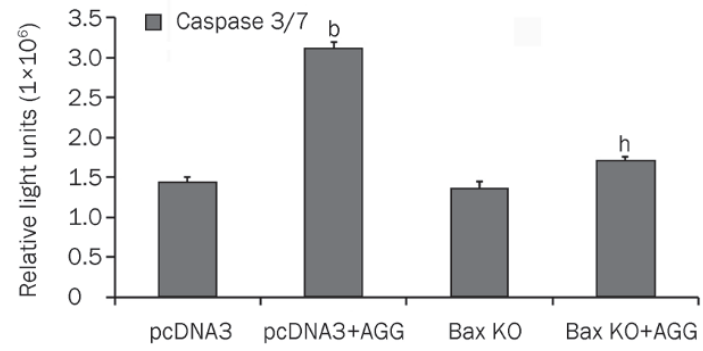

Figure 3. AGG induces a change in the apoptotic proteins in HepG2 cells. (A) HepG2 cells were treated with the indicated doses of AGG for $24 \mathrm{~h}$, and the cell lysates were immunoblotted with antibodies, as mentioned in 'Methods'. The protein band intensities of Bax (B), Bcl-2 (C) and cleaved PARP (CPARP) (D) were quantified using the ImageJ software with the obtained Western blots of the HepG2 cells. (E) The caspase-Glo 3/7, 8 and 9 activity assays were performed $24 \mathrm{~h}$ after AGG treatment with the indicated doses. (F) The HepG2 cells were pre-treated with 50 umol/L caspase 3 inhibitor (Cl) for $2 \mathrm{~h}$ prior to $\mathrm{AGG}(10 \mathrm{\mu g} / \mathrm{mL})$ exposure for a period of $24 \mathrm{~h}$, and the caspase $3 / 7$ activity was measured as previously mentioned. (G) The viability of the HepG2 cells was measured using a MTT assay with or without the prior addition of $\mathrm{Cl}$ (as mentioned above) in the AGG (10 $\mu \mathrm{g} / \mathrm{mL}$ )-exposed cells for $72 \mathrm{~h}$. (H) The caspase $3 / 7$ activity was also measured in a similar manner in the AGG-treated or untreated HepG2 cells that were transfected with pcDNA3 (mock) or a Bax KO vector, as mentioned in 'Methods'. All data are represented as the mean \pm SD of three different observations (using Student's $t$-test). ${ }^{\mathrm{b}} \mathrm{P}<0.05$ vs control, ${ }^{\mathrm{e}} \mathrm{P}<0.05$ vs AGG treated group and ${ }^{\mathrm{h}} \mathrm{P}<0.05$ vs mock transfected AGG treated group, were considered significant.

Hsp90 expression, which plays an important role in providing a survival advantage to tumor cells, decreased in a dose- oriented manner, as demonstrated by Western blotting analysis and immunocytofluorescence study (Figure 5A-5D). Simi- 
A
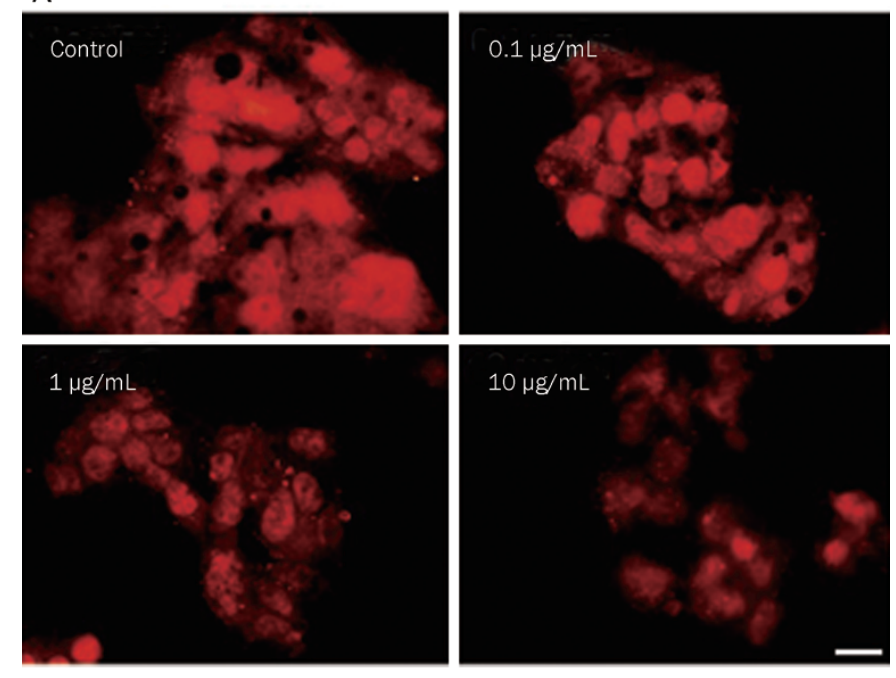

B

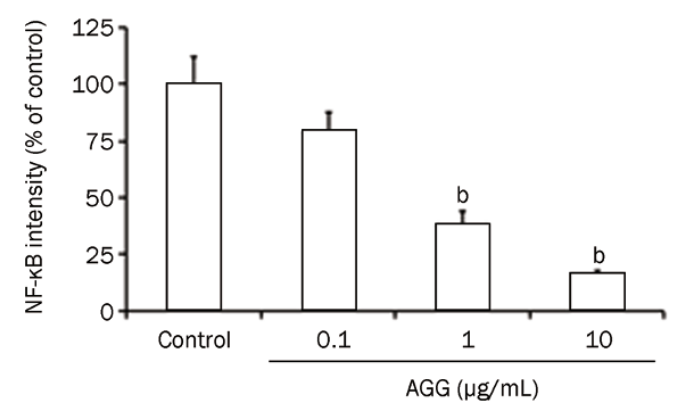

C

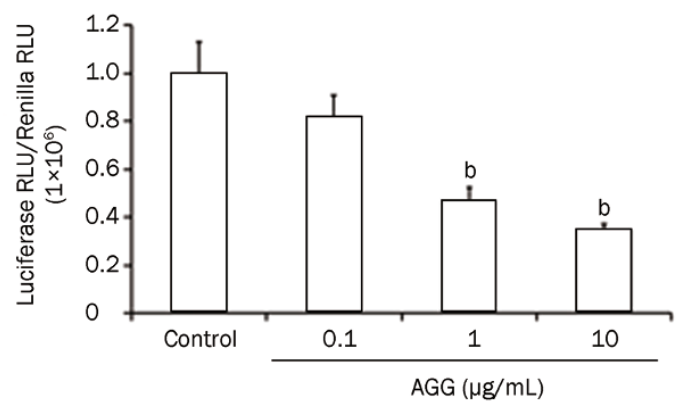

Figure 4. Effect of AGG on NF-KB expression. HepG2 cells were treated with $A G G$ for $12 \mathrm{~h}$, and the expression of NF-KB was analyzed by immunocytofluorescence $(A)$ and quantified using the ImageJ software (B). (C) HepG2 cells were transfected with a plasmid containing a NF-KBresponsive promoter that activates the transcription of firefly luciferase and a constitutively active Renilla luciferase plasmid to control for the transfection efficiency. These transfected cells were treated with the indicated AGG concentrations for $12 \mathrm{~h}$, and using cell lysates, the luminescence was determined using the Dual-Luciferase Reporter Assay with the help of the FLUOstar Optima Luminometer (BMG Labtech). The Relative Light Units (RLU) were obtained for both the Renilla and Firefly Luciferases, and all of the results are expressed as the ratio of Luciferase RLU to Renilla RLU. The scale bar represents $20 \mu \mathrm{m}$. The data are reported as the mean $\pm S D$ of three different observations. ${ }^{b} P<0.05$ vs PBS control. larly, AGG decreased the phosphorylation of Akt in a timedependent manner without affecting the total Akt expression (Figure 5E-5G).

AGG inhibits primary and distant tumors generated by liver cancer cells in nude mice

Finally, to demonstrate the in vivo efficacy of AGG in the human liver cancer xenograft model, HepG2 tumors were established in the flanks of athymic nude mice. After palpable tumors of $\sim 100 \mathrm{~mm}^{3}$ developed (in approximately 10-15 days) the animals received one injection per day in the peritoneum of $500 \mu \mathrm{g} / \mathrm{kg}$ body weight for 10 consecutive days. The control group developed large, aggressive and actively proliferating tumors in nude mice. However, the AGG-treated mice exhibited reduced tumor growth, demonstrating that the AGG-treated groups had significantly lower tumor weight and volume compared to the control group (Figure 6A and $6 \mathrm{~B})$. We initially began working with male athymic nude mice weighing approximately $25 \pm 2 \mathrm{~g}$, and did not observe any statistically significant changes in the body weight of the nude mice during the entire course of the experiment. Moreover, as shown in Figure 6A, the average tumor weight in the control group was $1.6 \pm 0.54 \mathrm{~g}$, which is not significantly different from that in the nude mice. The tumor tissues were subjected to immunohistochemistry for the cell proliferation marker Ki-67 and the angiogenic marker CD-31 and to an apoptotic TUNEL assay. In the HepG2 xenografts, the AGG-treated group showed a significant reduction in CD-31 and Ki-67 staining as well as enhanced TUNEL expression compared to the control (Figure 7). These findings indicate that AGG can efficiently regulate the inhibition of cell proliferation and angiogenesis, resulting in tumor growth inhibition.

\section{Discussion}

Lectins are naturally occurring potential alternative anticancerous therapeutic agents, and there have been several reports regarding the antineoplastic response due to various phytolectins $^{[24,25]}$. This Achilles heel approach supports lectins as principal precursors for therapeutic treatments. In addition to advanced lectin research in China and India, Europe has also begun to adopt lectins as alternative tumor therapies. The present work elucidated the antineoplastic potential of AGG, a RIP II family member that triggers caspase-mediated, apoptotic programmed type-I cell death. Here, we report for the first time the novel in vitro and in vivo effects of AGG on HCC.

The insight into the molecular machinery of lectin-mediated cell death has opened a new perception of complementary and alternative medicinal development. In this regard, several plant lectins, including Phaseolus vulgaris lectin, ConcanavalinA (ConA) and lectins from Astragalus membranaceus and Momordica charantia, induce apoptotic cell death in cancer cells that originate from different sources and tissues ${ }^{[26-30]}$. Additionally, ConA has been reported to elicit autophagic cell death in $\mathrm{HCC}^{[31]}$. In addition to this study, we have shown 
A



C
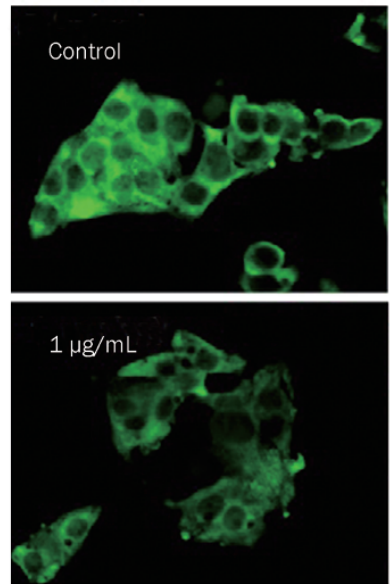

E
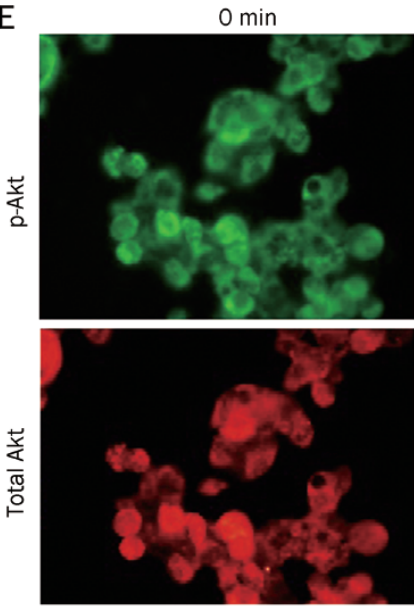

F

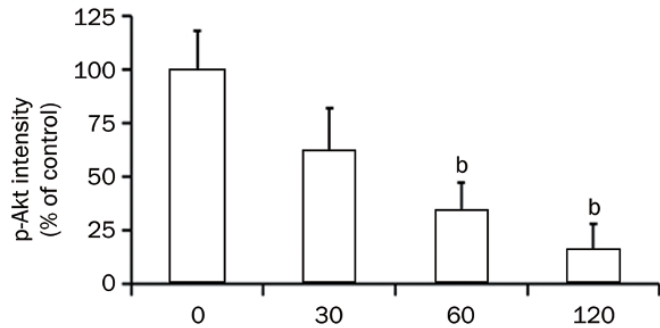

Duration of $10 \mu \mathrm{g} / \mathrm{mL}$ AGG treatment (min)
B

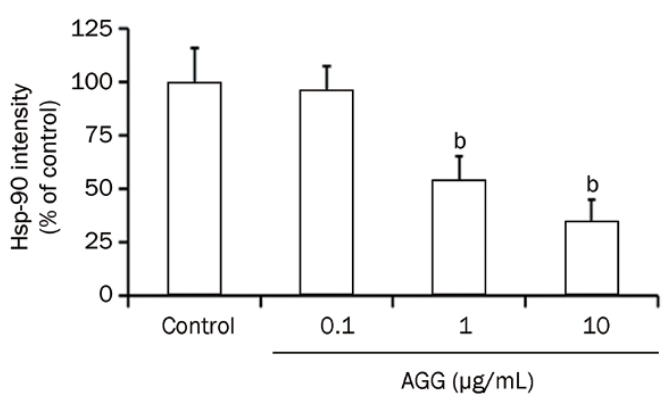

D

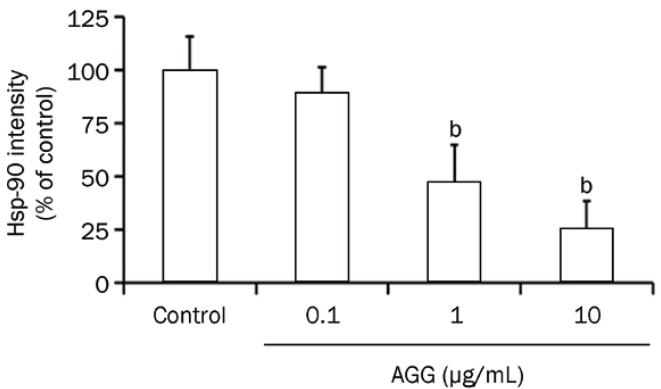

$60 \mathrm{~min}$
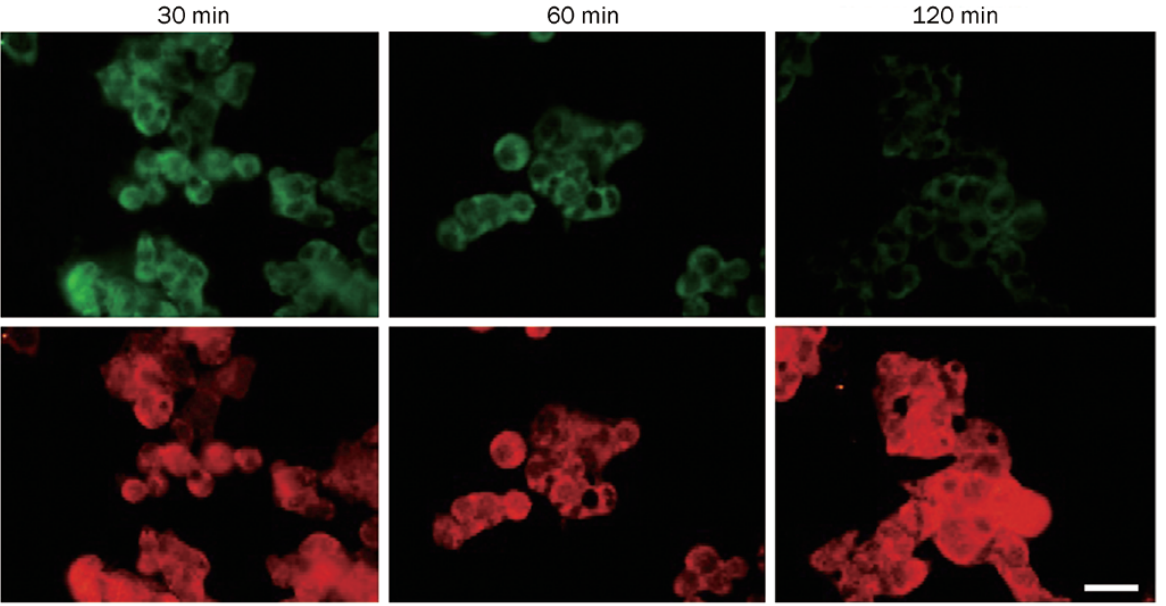

G

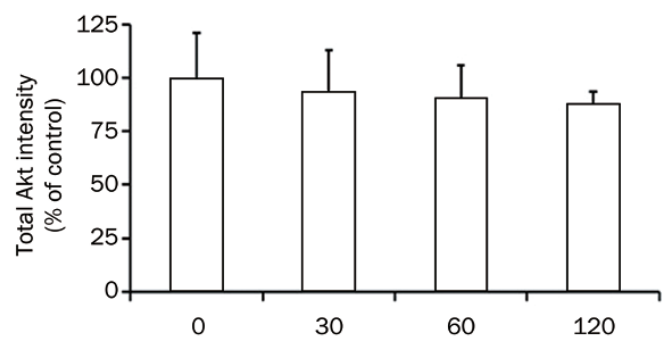

Duration of $10 \mu \mathrm{g} / \mathrm{mL}$ AGG treatment (min)

Figure 5. AGG reduces Hsp90 expression and suppresses Akt phosphorylation in HepG2 cells. The expression of Hsp90 was analyzed by Western blotting (A, B) and immunocytofluorescence (C, D) after $24 \mathrm{~h} \mathrm{AGG} \mathrm{treatment} \mathrm{at} \mathrm{the} \mathrm{indicated} \mathrm{doses.} \mathrm{The} \mathrm{HepG2} \mathrm{cells} \mathrm{were} \mathrm{treated} \mathrm{with} \mathrm{AGG} \mathrm{(10} \mu \mathrm{g} / \mathrm{mL})$ for different durations, and the expression of $p$-Akt and total Akt were analyzed by immunocytofluorescence (E-G). The scale bar represents $20 \mu m$. The data are reported as the mean \pm SD. ${ }^{b} \mathrm{P}<0.05 \mathrm{vs}$ control. All images and blots were quantified using the ImageJ software.

that AGG acts as an agent with a selective ability to induce cell death in various cancer cells without affecting the normal cells, presenting an ideal phyto-chemotherapeutic agent ${ }^{[7,9,32]}$. Huang et al reported a novel lectin from Astragalus membra- 


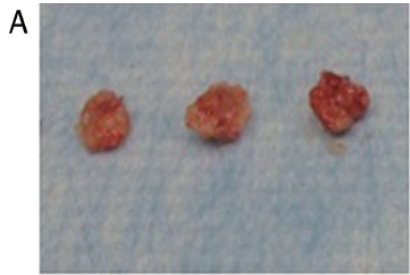

Control

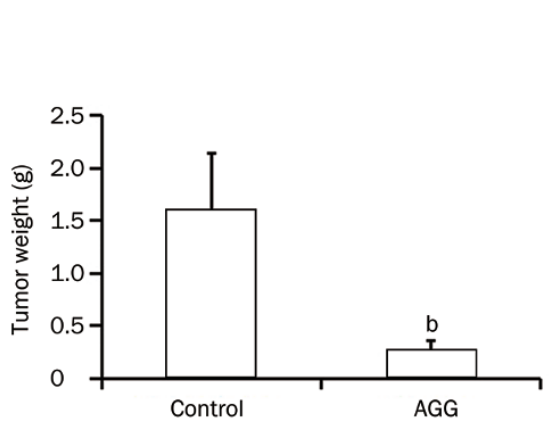

$\mathrm{B}$
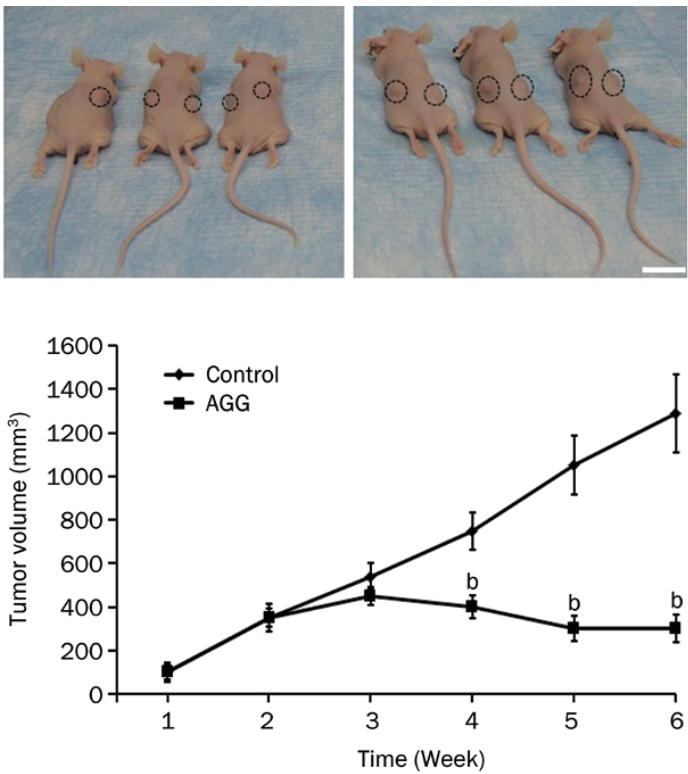

Figure 6. AGG inhibits tumor growth generated by liver cancer cells in nude mice. Tumor xenografts from the HepG2 cells were established in athymic nude mice in the flanks and were administered with PBS (control) or AGG (500 $\mu \mathrm{g} / \mathrm{kg}$ body weight) in an ip route for 10 consecutive days. (A) Photograph of the xenograft tumor with the measurement of tumor weight and (B) average measurement of the tumor xenograft volume (the tumor spots are circled in black rings), with a minimum of five mice in each group at the end of the study. The data are reported as the mean \pm SD. ${ }^{b} P<0.05$ vs control. The scale bar represents $15 \mathrm{~mm}$.

CD-31

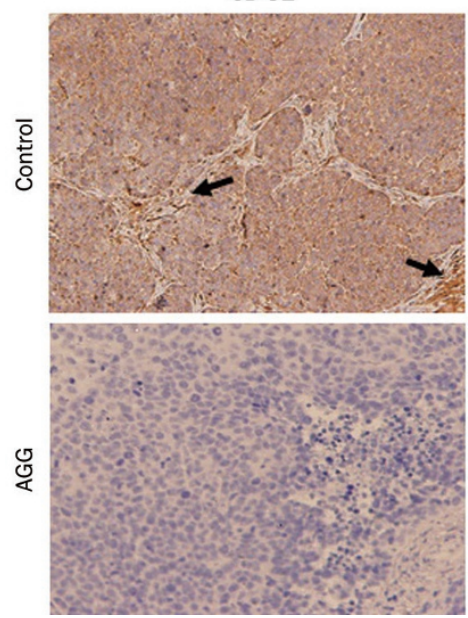

Ki-67

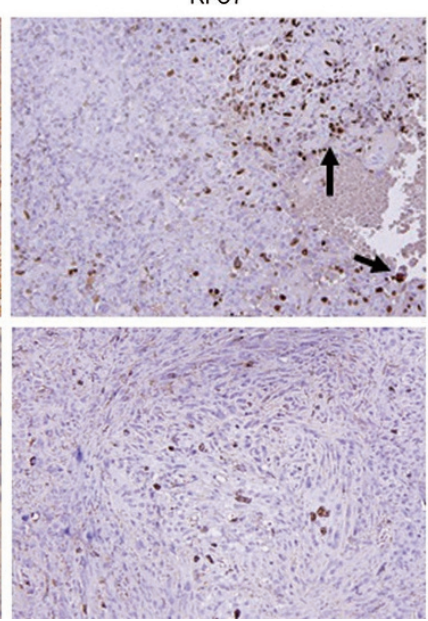

TUNEL

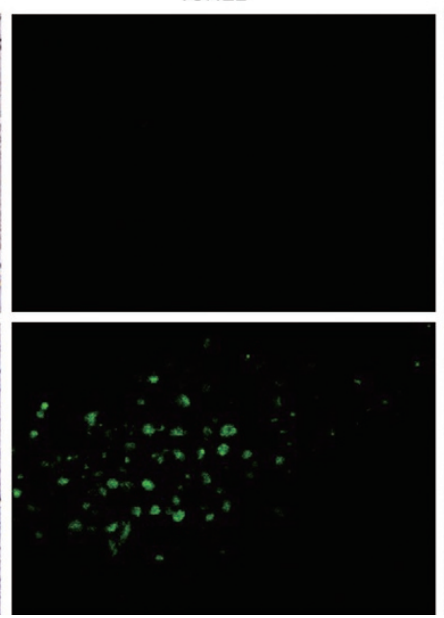

DAPI

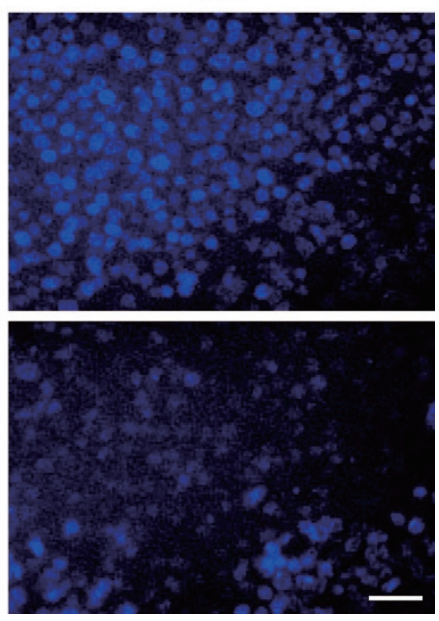

Figure 7. Immunohistochemical analysis of the tumor xenografts induced by HepG2 cells treated with AGG. The tumor tissues were harvested and fixed with formalin, and the following paraffin-embedded sections were immunostained: CD-31, Ki-67 (arrows indicate regions with high expression of CD-31 and Ki-67) and TUNEL as described in 'Methods'. The scale bar represents $20 \mu \mathrm{m}$.

naceus that induces caspase-dependent apoptosis in human leukemia cells ${ }^{[30]}$. The immunoblot analysis of AGG in our study revealed a sharp upregulation of Bax and a simultaneous downregulation of Bcl-2, along with PARP cleavage, in a dose-oriented manner. Interestingly, AGG failed to increase the caspase 3/7 activity level following the knockout of Bax in HepG2 cells, indicating that AGG-triggered caspase-mediated cell death is eventually increased along with Bax expression. By establishing a Bcl-2 over-expressing HCC cell line, Hu et al demonstrated that $\mathrm{Bcl}-2$ stimulates the survival of hepatocellular cells against plant lectin ricin insult ${ }^{[33]}$. The antineoplastic efficiency of Momordica charantia lectin against nasopharyngeal carcinoma progressed through cytochrome c released from damaged mitochondria followed by the activation of caspases 8, 9 and 3, thereby increasing the production of cleaved PARP and resulting in DNA fragmentation and apoptosis ${ }^{[31,32]}$.

Following AGG exposure, the Western blotting analysis demonstrated that the decrease in Hsp90 expression resulted 
in a decrease in the p-Akt level and a consequent decrease in the downstream NF- $\mathrm{kB}$ inflammatory expression and concomitant activation of caspases, leading to apoptosis. Qing et al previously reported the molecular link between Hsp90 inhibition and NF-KB downregulation through geldanamycin, a specific Hsp90 inhibitor ${ }^{[34]}$. Another study by Pandey et al demonstrated that Hsp90 overexpression inhibits apoptosis by interfering with Apaf-1 negatively ${ }^{[35]}$. The antiapoptotic function of Hsp90 is further demonstrated by its ability to interact with the p-Akt protein, which plays a pivotal role in cell survival by caspase 9 inactivation, thereby ensuring cellular survival $^{[36,37]}$. In this connection it was found that AGG reduces the Hsp90 level which might result in a downstream suppression of Akt phosphorylation. Interestingly, previous studies demonstrated that ConA regulates antineoplastic activity through the IKK-NF-KB-COX-2 pathway ${ }^{[38,39]}$.

In support of our previous findings ${ }^{[7,9,10,14]}$, this study showed that AGG significantly suppressed the growth of HepG2 tumors grafted onto the flank of nude mice, and reduced CD-31 and Ki-67 staining as well as enhanced TUNEL expression. These results indicate that AGG can efficiently regulate the inhibition of cell proliferation and angiogenesis, resulting in tumor growth inhibition. AGG was administered intraperitoneally to suppress the growth of distant tumors in the flank, indicating a potential association of AGG's immunoadjuvant and bystander activity in systemic tumor regres-

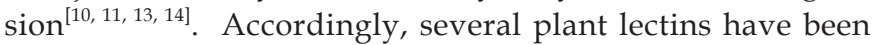
widely studied and were found to possess different tumor suppressive activities that are associated with a potent anticancer response ${ }^{[25,28,31]}$. For example, mistletoe lectins belonging to the RIP II family have been shown to inhibit lymphoma growth in mice and are used as an adjuvant therapy to treat various forms of human cancers ${ }^{[40]}$. Therefore, the results of this study support the use of AGG as a naturally occurring anticancer molecule for liver cancer treatment. Further insight into the molecular mechanisms involved in the apoptotic machinery will help us gain knowledge regarding the antineoplastic efficacy of AGG.

\section{Acknowledgements}

We thank the National Institute of Technology, Rourkela and Indian Institute of Technology, Kharagpur, for providing the facility for this research. This research was supported in part by the Rapid Grant for Young Investigator (RGYI) award, Department of Biotechnology, Government of India and Science and Engineering Research Board (SERB), Department of Science and Technology, Government of India. We also acknowledge the input from Debasree Ghosh and Abhishek NANDY, CSIR-SRF fellows of the CSIR-Indian Institute of Chemical Biology, Council of Scientific and Industrial Research, Kolkata, India, who helped to prepare the revised manuscript.

\section{Author contribution}

Subhadip MUKHOPADHYAY, Sujit Kumar BHUTIA and Tapas Kumar MAITI designed the research. Subhadip MUK-
HOPADHYAY, Sujit Kumar BHUTIA, Prashanta Kumar PANDA and Birendra BEHERA performed the experiments; Subhadip MUKHOPADHYAY, Durgesh Nandini DAS and Niharika SINHA analyzed the data; Subhadip MUKHOPADHYAY and Sujit Kumar BHUTIA wrote the manuscript.

\section{Supplementary information}

Supplementary information is available at Acta Pharmacologica Sinica's website.

\section{References}

1 El-Serag HB, Davila JA. Surveillance for hepatocellular carcinoma: in whom and how? Therap Adv Gastroenterol 2011; 4: 5-10.

2 Kim HY, Park JW, Nam BH, Kim HK, Choi Jl, Kim TH, et al. Survival of patients with advanced hepatocellular carcinoma: sorafenib versus other treatments. J Gastroenterol Hepatol 2011; 26: 1612-8.

3 Jemal A, Siegel R, Ward E, Hao Y, Xu J, Murray T, Thun MJ. Cancer statistics, 2008. CA Cancer J Clin 2008; 58: 71-96.

4 Leong TY, Leong AS. Epidemiology and carcinogenesis of hepatocellular carcinoma. HPB (Oxford) 2005; 7: 5-15.

5 El-Serag HB, Rudolph KL. Hepatocellular carcinoma: epidemiology and molecular carcinogenesis. Gastroenterology 2007; 132: 255776.

6 Rogers AB, Theve EJ, Feng Y, Fry RC, Taghizadeh K, Clapp KM, et al. Hepatocellular carcinoma associated with liver-gender disruption in male mice. Cancer Res 2007; 67: 11536-46.

7 Bhutia SK, Mallick SK, Stevens SM, Prokai L, Vishwanatha JK, Maiti TK. Induction of mitochondria-dependent apoptosis by Abrus agglutinin derived peptides in human cervical cancer cell. Toxicol In Vitro 2008; 22: 344-51.

8 Hegde R, Maiti TK, Podder SK. Purification and characterization of three toxins and two agglutinins from Abrus precatorius seed by using lactamyl-Sepharose affinity chromatography, Anal Biochem 1991; 194: 101-9.

9 Bhutia SK, Mallick SK, Maiti S, Maiti TK. Antitumor and proapoptotic effect of Abrus agglutinin derived peptide in Dalton's lymphoma tumor model. Chem Biol Interact 2008; 174: 11-8.

10 Ghosh D, Bhutia SK, Mallick SK, Banerjee I, Maiti TK. Stimulation of murine $B$ and $T$ lymphocytes by native and heat-denatured Abrus agglutinin. Immunobiology 2009; 214: 227-34.

11 Tripathi S, Maiti TK. Efficiency of heat denatured lectins from Abrus precatorius as immunoadjuvants. Food Agric Immunol 2003; 15: 279-87.

12 Tripathi S, Maiti TK. Stimulation of murine macrophages by native and heat-denatured lectin from Abrus precatorius. Int Immunopharmacol 2003; 3: 375-81.

13 Tripathi S, Maiti TK. Immunomodulatory role of native and heat denatured agglutinin from Abrus precatorius. Int J Biochem Cell Biol 2005; 37: 451-62.

14 Bhutia SK, Mallick SK, Maiti TK. In vitro immunostimulatory properties of Abrus lectins derived peptides in tumor bearing mice. Phytomedicine 2009; 16: 776-82.

15 Bhutia SK, Dash R, Das SK, Azab B, Su ZZ, Lee SG, et al. Mechanism of autophagy to apoptosis switch triggered in prostate cancer cells by antitumor cytokine melanoma differentiation-associated gene 7/ interleukin-24. Cancer Res 2010; 70: 3667-76.

16 Mukhopadhyay S, Panda PK, Behera B, Das CK, Hassan MK, Das $\mathrm{DN}$, et al. In vitro and in vivo antitumor effects of Peanut agglutinin through induction of apoptotic and autophagic cell death. Food Chem Toxicol 2014; 64: 369-77. 
17 Bhutia SK, Kegelman TP, Das SK, Azab B, Su ZZ, Lee SG, et al. Astrocyte elevated gene-1 induces protective autophagy. Proc Natl Acad Sci U S A 2010; 107: 22243-8.

18 Zhang L, Yu J, Park BH, Kinzler KW, Vogelstein B. Role of BAX in the apoptotic response to anticancer agents. Science 2000; 290: 98992.

19 Boyd JH, Kan B, Roberts H, Wang Y, Walley KR. S100A8 and S100A9 mediate endotoxin-induced cardiomyocyte dysfunction via the receptor for advanced glycation end products. Circ Res 2008; 102: 1239-46.

20 Schneider CA, Rasband WS, Eliceiri KW. NIH Image to ImageJ: 25 years of image analysis. Nat Methods 2012; 9: 671-5.

21 Bhutia SK, Das SK, Kegelman TP, Azab B, Dash R, Su ZZ, et al. mda7/IL-24 differentially regulates soluble and nuclear clusterin in prostate cancer. J Cell Physiol 2011; 227: 1805-13.

22 Chaitanya GV, Steven AJ, Babu PP. PARP-1 cleavage fragments: signatures of cell-death proteases in neurodegeneration. Cell Commun Signal 2010; 8: 31.

23 Dolcet X, Llobet D, Pallares J, Matias-Guiu X. NF-kB in development and progression of human cancer. Virchows Arch 2005; 446: 47582.

24 Bhutia SK, Maiti TK. Targeting tumors with peptides from natural sources. Trends Biotechnol 2008; 26: 210-7.

25 Liu B, Bian HJ, Bao JK. Plant lectins: potential antineoplastic drugs from bench to clinic. Cancer Lett 2010; 287: 1-12.

26 Fang EF, Pan WL, Wong JH, Chan YS, Ye XJ, et al. A new Phaseolus vulgaris lectin induces selective toxicity on human liver carcinoma HepG2 cells. Arch Toxicol 2011; 85: 1551-63.

27 Liu B, Li CY, Bian HJ, Min MW, Chen LF, Bao JK. Antiproliferative activity and apoptosis-inducing mechanism of Concanavalin A on human melanoma A375 cells. Arch Biochem Biophys 2009; 482: 1-6.

28 Fang EF, Zhang CZ, Ng TB, Wong JH, Pan WL, Ye XJ, et al. Momordica Charantia lectin, a type II ribosome inactivating protein, exhibits antitumor activity toward human nasopharyngeal carcinoma cells in vitro and in vivo. Cancer Prev Res (Phila) 2012; 5: 109-21.

29 Li CJ, Tsang SF, Tsai CH, Tsai HY, Chyuan JH, Hsu HY. Momordica charantia extract induces apoptosis in human cancer cells through caspase- and mitochondria-dependent pathways. Evid Based Complement Alternat Med 2012; 261971.
30 Huang LH, Yan QJ, Kopparapu NK, Jiang ZQ, Sun Y. Astragalus membranaceus lectin (AML) induces caspase-dependent apoptosis in human leukemia cells. Cell Prolif 2012; 45: 15-21.

31 Chang CP, Yang MC, Liu HS, Lin YS, Lei HY. Concanavalin A induces autophagy in hepatoma cells and has a therapeutic effect in a murine in situ hepatoma model. Hepatology 2007; 45: 286-96.

32 Bhutia SK and Maiti TK. Crabs Eye (Abrus precatorius) seed and its immunomodulatory and antitumor properties. In: Preedy VR, Watson RR, Patel VB editors. Nuts and seeds in Health and Disease Prevention. Academic Press; 2011. p 409-15.

33 Hu R, Zhai Q, Liu W, Liu X. An insight into the mechanism of cytotoxicity of ricin to hepatoma cell: roles of Bcl-2 family proteins, caspases, $\mathrm{Ca}(2+)$-dependent proteases and protein kinase C. J Cell Biochem 2001; 81: 583-93.

34 Qing G, Yan P, Xiao G. Hsp90 inhibition results in autophagy-mediated proteasome-independent degradation of IkappaB kinase (IKK). Cell Res 2006; 16: 895-901.

35 Pandey P, Saleh A, Nakazawa A, Kumar S, Srinivasula SM, Kumar V, et al. Negative regulation of cytochrome c-mediated oligomerization of Apaf-1 and activation of procaspase- 9 by heat shock protein 90 . EMBO J 2000; 19: 4310-22.

36 Sato S, Fujita N, Tsuruo T. Modulation of Akt kinase activity by binding to Hsp90. Proc Natl Acad Sci U S A 2000; 97: 10832-7.

37 Bai L, Xu S, Chen W, Li Z, Wang X, Tang H, et al. Blocking NF-KB and Akt by Hsp90 inhibition sensitizes Smac mimetic compound 3 -induced extrinsic apoptosis pathway and results in synergistic cancer cell death. Apoptosis 2011; 16: 45-54.

38 Li WW, Yu JY, Xu HL, Bao JK. Concanavalin A: a potential antineoplastic agent targeting apoptosis, autophagy and anti-angiogenesis for cancer therapeutics. Biochem Biophys Res Commun 2011; 414: 282-6.

39 Sina A, Proulx-Bonneau S, Roy A, Poliquin L, Cao J, Annabi B. The lectin concanavalin-A signals MT1-MMP catalytic independent induction of COX-2 through an IKKY/NF-KB-dependent pathway. J Cell Commun Signal 2010; 4: 31-8.

40 Seifert G, Jesse P, Laengler A, Reindl T, Lüth M, Lobitz S, et al. Molecular mechanisms of mistletoe plant extract-induced apoptosis in acute lymphoblastic leukemia in vivo and in vitro. Cancer Lett 2008; 264: 218-28. 